\title{
POSITIVE SOLUTIONS FOR NONLINEAR PARABOLIC SECOND INITIAL BOUNDARY VALUE PROBLEMS*
}

\author{
$\mathrm{BY}$ \\ C. Y. CHAN \\ Florida State University
}

1. Introduction. Existence and uniqueness of the positive solution for a nonlinear second initial boundary value problem involving a one-dimensional heat equation with zero initial distribution of temperature and a nonlinear radiation boundary condition were established by Mann and Wolf [11]. Their results were improved by Roberts and Mann [16] and Padmavally [14]. Recently, Keller and Olmstead [10] gave a constructive proof of the existence for a problem of this type. Using Schauder's fixedpoint theorem [18], Friedman [5] considered an $n$-dimensional linear parabolic equation with linear initial and nonlinear second boundary conditions. The maximum principle [6, pp. $34-40 ; 12 ; 15$, pp. 173-175] was used to prove uniqueness, and a constructive proof of the existence was given by Chan [2] for a problem consisting of an $n$-dimensional semilinear heat equation under linear initial and nonlinear radiation boundary conditions with the use of the variational properties of the Neumann functions. In these last two papers, the solutions need not be positive.

The purpose of this paper is to establish uniqueness, existence, upper and lower bounds of positive solutions for a class of nonlinear second initial boundary value problems more general than that considered by Chan [2]. The techniques used are different from those in the above-mentioned papers. Our class of problems consists of a semilinear parabolic equation under linear initial and nonlinear radiation boundary conditions. Positive steady-state solutions for problems of this type were considered by Olmstead [13], Keller [9], Cohen and Laetsch [4], and more recently by Cohen [3].

In Sec. 2 we establish uniqueness of a solution (not necessarily positive) under less stringent conditions than those imposed by Chan [2]. Conditions which imply that the solution is positive are given in Theorem 2 . An existence theorem of the positive solution is proved constructively in Sec. 3 by using an iteration scheme of the Picard type. This scheme gives an alternating sequence consisting of two monotone subsequences bounding the solution from above and below. Thus in a given problem, each successive iteration yields a more accurate pointwise upper or lower bound. The sequence is shown to converge geometrically to obtain the existence theorem. In Sec. 4 we first use the quasilinearization technique to establish an existence theorem. We show that this technique gives a monotone non-increasing sequence, converging quadratically to the solution. The method of quasilinearization was introduced in dynamic programming by Bellman [1]. It was used by Keller [9] and more recently by Cohen [3] for some $n$-dimensional mildly nonlinear elliptic boundary-value problems. To obtain the lower bounds, we

\footnotetext{
* Received July 8, 1972; revised version received October 25, 1972.
} 
construct a monotone nondecreasing sequence converging to the solution. From these constructions, pointwise upper and lower bounds are also obtained.

2. Uniqueness. Let $l$ be a bounded $n$-dimensional domain in the real $n$-dimensional Euclidean space, $D^{-}$its closure, and $\partial D$ its boundary. Also let $x=\left(x_{1}, x_{2}, \cdots, x_{n}\right)$, $\Omega=D \times(0, T], T<\infty$, and $S=\partial D \times(0, T]$. The semilinear parabolic equation under consideration is

$$
L u \equiv \sum_{i, j=1}^{n} \frac{\partial}{\partial x_{i}}\left\{a_{i j}(x, t) \frac{\partial u}{\partial x_{i}}\right\}+c(x, t) u-\frac{\partial u}{\partial t}=g(x, t ; u) \text { in } \Omega,
$$

where $c \leq 0$ is continuous, $a_{i j}=a_{i i}(i, j=1,2, \cdots, n)$ are continuously differentiable, and for all $n$-tuples of real numbers $\left(\xi_{1}, \xi_{2}, \cdots, \xi_{n}\right)$, there exists a positive number $\kappa$ such that

$$
\sum_{i, j=1}^{n} a_{i} \xi_{i} \xi_{i} \geq \kappa \sum_{i=1}^{n} \xi_{i}{ }^{2}
$$

for all $(x, t)$ in $\Omega$. Let $B_{\tau}=D \times[0, T] \cap\{t=\tau\}$. The initial condition is given by

$$
u(., 0)=\phi(x) \text { on } B_{0}{ }^{-} .
$$

Let $n=\left(n_{1}, n_{2}, \cdots, n_{n}\right)$ be the outward unit normal to $\partial D$. Our nonlinear radiation boundary condition is given by

$$
A u \equiv \frac{\partial u}{\partial \nu(x, t)}+B(x, t ; u)=f(x, t) \quad \text { on } S,
$$

where $\partial / \partial \nu \equiv \sum_{i, j=1}^{n} a_{i j} n_{i}\left(\partial / \partial x_{i}\right)$ is the outward conormal derivative to $S$.

For $n=3, a_{i j}=1$ if $i=j$, and $a_{i j}=0$ if $i \neq j$, the problem (2.1)-(2.3) can be interpreted physically as that of finding the temperature $u(x, t)$ of a homogeneous and isotropic solid having an arbitrary initial distribution of temperature $\phi(x)$. Heat is generated nonlinearly in it at a rate proportional to $c u-g$, and the body is subject to a nonlinear radiation boundary condition (2.3), which is more general than the Stefan fourth-power law [13]. Our quest for positive solutions is motivated by the physical concept of the absolute temperature.

Let the subregions $D \times(\tau, t]$ and $\partial D \times(\tau, t]$ be denoted respectively by $\Omega_{\tau t}$ and $S_{\tau t}$.

Theorem 1. Let $M(x, t)$ satisfy $L M \leq g(x, t ; M)$ in $\Omega, M(x, 0) \geq \phi(x)$ on $B_{0}{ }^{-}$, $A M \geq f$ on $S$. Also let $m(x, t)$ satisfy $\operatorname{Lm} \geq g(x, t ; m)$ in $\Omega, m(x, 0) \leq \phi(x)$ on $B_{0}{ }^{-}$, $A m \leq f$ on $S$. If $m, u$ and $M$ are continuous on $\Omega^{-}$where $u$ is a solution of the problem (2.1)-(2.3), and if

$$
\begin{aligned}
& g(\xi, \tau ; \zeta(\xi, \tau)) \geq g(\xi, \tau ; z(\xi, \tau)) \text { when } \zeta(\xi, \tau)>z(\xi, \tau), \\
& B(\xi, \tau ; \zeta(\xi, \tau))>B(\xi, \tau ; z(\xi, \tau)) \text { when } \zeta(\xi, \tau)>z(\xi, \tau) \text {, }
\end{aligned}
$$

then $m(x, t) \leq u(x, t) \leq M(x, t)$ on $\Omega^{-}$.

Proof. Let $w=M-u$. If $w<0$ at some point of $\Omega^{-}$, then since $w$ is continuous on $\Omega^{-}, w$ attains its negative minimum $c_{1}$ at some point, say $(x, t)$. If $(x, t)$ is in $\Omega_{0 t}$, then let $\omega$ be the largest subset of $\Omega_{0,}$ such that $w<0$. From (2.4), Lw $\leq 0$ in $\omega$, and hence by the strong maximum principle $v \equiv c_{1}$ in $\omega$, contradicting the definition of $\omega$ 
unless $\omega=\Omega_{0 t}$. But this latter case contradicts by continuity the condition $w \geq 0$ on $B_{0}{ }^{-}$. Therefore the negative minimum cannot be in $\Omega_{0 t}$. If $(x, t)$ is on $S_{0 t}$, then at this point $\partial w / \partial \nu \leq 0$, and $B(x, t ; M)-B(x, t ; u)<0$ by (2.5). This contradicts the given condition $A M-A u \geq 0$. Thus $w \geq 0$ on $\Omega^{-}$, and hence $M \geq u$ on $\Omega^{-}$.

To prove $m \leq u$ on $\Omega^{-}$, we let $z=u-m$ and use a similar argument to conclude $z \geq 0$ on $\Omega^{-}$. Thus the theorem is proved.

From this theorem, we obtain uniqueness of the solution.

Corollary 1. If (2.4) and (2.5) hold, then there exists at most one solution to the problem (2.1)-(2.3).

Theorem 2. If there exists a positive constant $c_{2}$ such that $u>v$ at the point $(\xi, \tau)$ implies

and if

$$
c_{2}\{u(\xi, \tau)-v(\xi, \tau)\} \geq g(\xi, \tau ; u(\xi, \tau))-g(\xi, \tau ; v(\xi, \tau)),
$$

$$
\begin{aligned}
g(x, t ; 0) & =0, & & \phi(x)>0, \\
B(x, t ; 0) & =0, & & f(x, t)>0,
\end{aligned}
$$

and if (2.4) and (2.5) hold, then there exists at most one solution $u$ of the problem (2.1)-(2.3); if a solution exists, it is positive.

Proof. It follows from Corollary 1 that it is sufficient to show $u>0$ on $\Omega^{-}$. Let $m(x, t) \equiv 0$ on $\Omega^{-}$. Then $L m=0=g(x, t ; m)$ in $\Omega, m=0<\phi(x)$ on $B_{0}{ }^{-}, A m=0<f$ on $S$. Hence, by Theorem $1, u(x, t) \geq 0$ on $\Omega^{-}$.

From (2.1) and (2.7), we have $L(u-0)=g(x, t ; u)-g(x, t ; 0)$. Thus, from (2.6), $\left(L-c_{2}\right) u \leq 0$ in $\Omega$. By (2.8), $u>0$ on $B_{0}{ }^{-}$. If $u \leq 0$ at some point of $\Omega^{-}$, then, by the weak maximum principle, $u$ attains its non-positive minimum at some point on $S$. It follows from (2.5) and (2.9) that $A u \leq 0$ at this point. This contradicts (2.10). Thus $u>0$ on $\Omega^{-}$.

3. Iteration scheme of the Picard type. Let us give the following definitions:

Definition 1. A function $k(x)$ is said to belong to the class $C^{m+\alpha}$ if all its first $m$ partial derivatives exist, are continuous and are locally Hölder-continuous of exponent $\alpha$, where $0<\alpha<1$.

Definition 2. The boundary $\partial D$ belongs to the class $C^{m+\alpha}$ if for every point $x$ of $\partial D$, there exists an $n$-dimensional neighborhood $K$ such that $K \cap \partial D$ can be represented for some $i(1 \leq i \leq n)$ in the form $x_{i}=h\left(x_{1}, x_{2}, \cdots, x_{i-1}, x_{i+1}, \cdots, x_{n}\right)$, where $h$ belongs to $C^{m+\alpha}$.

We shall also need the following assumption:

(A) the coefficients $a_{i j}(i, j=1,2, \cdots, n)$, their partial derivatives $\partial a_{i j} / \partial x_{k}$, and $c$ are uniformly Hölder-continuous of exponent $\alpha$ on $\Omega^{-}$.

For convenience we state the following lemma whose proof can be found in Friedman [6, p. 146].

Lemma 1. Under assumptions (A) and $\partial D \in C^{1+\alpha}$, if $w$ is a solution of the problem $L w=b(x, t)$ in $\Omega, w=l(x)$ on $B_{0}^{-}$,

$$
\psi_{\lambda} w \equiv\left(\frac{\partial}{\partial \nu}+\lambda\right) w=p(x, t) \quad \text { on } \quad S,
$$


where $\lambda(x, t)$ is continuous on $S^{-}$, then for all $(x, t)$ on $\Omega^{-}$,

$$
\left.|w(x, t)| \leq \underset{\mathbf{8}^{-}}{c_{3}(\text { l.u.b. }}|b|+\underset{B_{0^{-}}}{\text {l.u.b. }}|l|, \underset{S^{-}}{\text {l.u.b. }}|p|\right)
$$

where $c_{3}$ is a constant depending on $L, \lambda$ and $\Omega^{-}$.

Let $\Omega^{*}=D \times[0, T), \Omega_{\tau t}{ }^{*}=D \times[\tau, t)$, and $S_{\tau t}{ }^{*}=\partial D \times[\tau, t)$. To define a Neumann function, we follow Friedman [6, p. 155].

Definition 3. A function $R(x, t ; \xi, \tau)$ defined and continuous for $(x, t ; \xi, \tau) \in \Omega^{-} \times \Omega^{*}$, $t>\tau$ is called a Neumann function of $L w=0$ in $\Omega$ and $\psi_{\beta} w=0$ on $S$, where $\beta(x, t)$ is continuous on $S^{-}$, if for any $0 \leq \tau<T$ and for any continuous function $l(x)$ on $B_{\tau}$ having a compact support, the function

$$
w=\int_{B_{\tau}} R(x, t ; \xi, \tau) l(\xi) d V_{\xi}
$$

is a solution of $L w=0$ in $\Omega_{\tau T}$, and satisfies

$$
\lim _{t \downarrow \tau} w(x, t)=l(x) \text { for } x \in B_{\tau}^{-},
$$

and $\psi_{\beta} w=0$ on $S_{\tau T}$.

Let $R^{*}(x, t ; \xi, \tau)$ denote the Neumann function of the adjoint equation $L^{*} w=0$ in $\Omega^{*}$ corresponding to the boundary condition $\psi_{\beta} w=0$ on $S_{0 \tau}{ }^{*}$. Under assumptions (A) and $\partial D \in C^{2+\alpha}$, it follows from Friedman [6, p. 155, pp. 82-84] and Itô [4, 5] that $R$ and $R^{*}$ exist and are unique, $L R=0$ for $(x, t)$ in $\Omega, L^{*} R^{*}=0$ for $(x, t)$ in $\Omega^{*}, \psi_{\beta} R=0$ for $(x, t)$ on $S_{\tau}, \psi_{\beta} R^{*}=0$ for $(x, t)$ on $S_{0 \tau}{ }^{*}$, and furthermore, $R, R_{x}, R_{x x}$ and $R_{t}$ are continuous functions of $(x, t ; \xi, \tau)$ in $\Omega \times \Omega^{*}, t>\tau$ while $R^{*}, R_{x}{ }^{*}, R_{x x}{ }^{*}$ and $R_{t}{ }^{*}$ are continuous functions of $(x, t ; \xi, \tau)$ in $\Omega^{*} \times \Omega, t<\tau$.

Let $\Gamma(x, t ; \xi, \tau)$ denote the fundamental solution of $L$. It can be constructed by the parametrix method [6, pp. 3-25]. Let $V(x, t ; \xi, \tau)$ denote the solution of the linear second initial boundary value problem: $L V=0$ in $\Omega_{\tau T}, V=0$ on $B_{\tau}^{-}, \psi_{\beta} V=-\psi_{\beta} \Gamma$ on $S_{\tau T}$. Then the Neumann function is given by

$$
R(x, t ; \xi, \tau)=\Gamma(x, t ; \xi, \tau)+V(x, t ; \xi, \tau) .
$$

By Friedman [6, p. 134],

$$
|\Gamma(x, t ; \xi, \tau)| \leq c_{4} /\left\{(t-\tau)^{\mu}|x-\xi|^{n-2 \mu}\right\} \equiv q(x-\xi, t-\tau)
$$

where $c_{4}$ is a positive constant and $0<\mu<1$. From Lemma $1,|V| \leq c_{5}$ on $\Omega^{-}$, where $c_{5}$ is a constant.

In the Green's identity

$$
v L u-u L^{*} v=\sum_{i=1}^{n} \frac{\partial}{\partial x_{i}}\left\{\sum_{i=1}^{n}\left(v a_{i j} \frac{\partial u}{\partial x_{i}}-u a_{i j} \frac{\partial v}{\partial x_{i}}\right)\right\}-\frac{\partial}{\partial t}(u v),
$$

let $u(y, \sigma)=R(y, \sigma ; \xi, \tau)$ and $v(y, \sigma)=R^{*}(y, \sigma ; x, t)$. Integrating this over the domain $D \times(\tau+\epsilon, t-\epsilon)$ and letting $\epsilon \rightarrow 0$, we have by the boundary condition

$$
R(x, t ; \xi, \tau)=R^{*}(\xi, \tau ; x, t)
$$

for any two points $(x, t)$ and $(\xi, \tau)$ in $\Omega$ with $t>\tau$. Using an argument similar to the proof of Theorem 11 of [6, pp. 44-45], we have for $(\xi, \tau)$ in $\Omega^{*}$

$$
R(x, t ; \xi, \tau)>0 \text { in } \Omega_{\tau} .
$$


Let $N(x, t ; \xi, \tau)$ be the Neumann function of $L w=0$ in $\Omega$ and $\partial w / \partial \nu=0$ on $S$.

Our iteration scheme is given by

$$
\begin{gathered}
L u_{0}=0 \text { in } \Omega, \\
u_{0}(x, 0)=\phi(x) \text { on } B_{0}^{-}, \\
\frac{\partial u_{0}}{\partial \nu}=f(x, t) \quad \text { on } S,
\end{gathered}
$$

and for $i=0,1,2, \cdots$,

$$
\begin{gathered}
L u_{i+1}=g\left(x, t ; u_{i}\right) \quad \text { in } \Omega, \\
u_{i+1}(x, 0)=\phi(x) \quad \text { on } B_{0^{-}} \\
\left(\partial u_{i+1} / \partial \nu\right)+B\left(x, t ; u_{i}\right)=f(x, t) \quad \text { on } S .
\end{gathered}
$$

Under assumptions $(A)$ and $\partial D \in C^{2+\alpha}, N(x, t ; \xi, \tau)$ exists, and hence the sequence $\left\{u_{i}\right\}$ is well defined, provided $g, \phi, B$ and $f$ are continuous. In the following theorem we show that this scheme gives an alternating sequence consisting of two monotone subsequences bounding the solution from above and below.

Theorem 3. Under assumptions $(A)$ and $\partial D \in C^{2+\alpha}$, and the hypotheses of Theorem 2 with (2.4) replaced by

$$
g(\xi, \tau ; \zeta(\xi, \tau))>g(\xi, \tau ; z(\xi, \tau)) \text { when } \zeta(\xi, \tau)>z(\xi, \tau),
$$

if $g, \phi, B$ and $f$ are continuous, then the solution $u$ of the problem (2.1)-(2.3) satisfies

$$
-c_{6} \leq u_{1}<\cdots<u_{2 i+1}<\cdots<u<\cdots<u_{2 i}<\cdots<u_{0} \leq c_{6} \text { in } \Omega,
$$

where $c_{6}=\max \left\{\right.$ l.u.b. $\Omega^{-} u_{0}$, l.u.b. $\left.\Omega^{-}\left|u_{1}\right|\right\}$.

Proof. Let $v=N^{*}(\xi, \tau ; x, t)$ in the Green's identity, and integrate over $D \times(\epsilon, t-\epsilon)$. On letting $\epsilon \rightarrow 0$ and using (3.1), we can rewrite (2.1)-(2.3) equivalently as

$$
\begin{aligned}
u(x, t)= & \int_{D} N(x, t ; \xi, 0) \phi(\xi) d V_{\xi}-\int_{0}^{t} \int_{D} N(x, t ; \xi, \tau) g(\xi, \tau ; u) d V_{\xi} d \tau \\
& +\int_{0}^{t} \int_{\partial D} N(x, t ; \xi, \tau)[f(\xi, \tau)-B(\xi, \tau ; u)] d A_{\xi} d \tau .
\end{aligned}
$$

Since $L\left(u_{0}-u\right)=-g(x, t ; u)$ in $\Omega, u_{0}-u=0$ on $B_{0}{ }^{-}$, and $(\partial / \partial \nu)\left(u_{0}-u\right)=$ $B(x, t ; u)$ on $S$, we have

$$
\begin{aligned}
u_{0}-u= & \int_{0}^{t} \int_{D} N(x, t ; \xi, \tau) g(\xi, \tau ; u) d V_{\xi} d \tau \\
& +\int_{0}^{t} \int_{\partial D} N(x, t ; \xi, \tau) B(\xi, \tau ; u) d A_{\xi} d \tau .
\end{aligned}
$$

By Theorem $2, u>0$ on $\Omega^{-}$. From (2.7) and (3.9), $g(\xi, \tau ; u)>0$. Similarly, from (2.5) and $(2.9), B(\xi, \tau ; u)>0$. By $(3.2), N(x, t ; \xi, \tau)>0$ in $\Omega_{\tau T}$. Thus the right-hand-side of (3.12) is positive, and hence $u_{0}>u$ in $\Omega$.

Since $u_{0}>u$ in $\Omega$, we have

$$
L\left(u-u_{1}\right)=g(x, t ; u)-g\left(x, t ; u_{0}\right)<0 \text { in } \Omega
$$


by (3.9). By continuity, $u_{0} \geq u$ on $S$. Hence it follows from (2.5) that $(\partial / \partial \nu)\left(u-u_{1}\right)=$ $B\left(x, t ; u_{0}\right)-B(x, t ; u) \geq 0$. From (3.11),

$$
\begin{aligned}
u-u_{1}= & \int_{0}^{t} \int_{D} N(x, t ; \xi, \tau)\left[g\left(\xi, \tau ; u_{0}\right)-g(\xi, \tau ; u)\right] d V_{\xi} d \tau \\
& +\int_{0}^{t} \int_{\partial D} N(x, t ; \xi, \tau)\left[B\left(\xi, \tau ; u_{0}\right)-B(\xi, \tau ; u)\right] d A_{\xi} d \tau>0 .
\end{aligned}
$$

Thus $u>u_{1}$ in $\Omega$.

From Lemma 1,

$$
\begin{aligned}
& u_{0} \leq c_{3}\left(\underset{B_{0}-}{\text { l.u.b. } \phi}+\underset{s^{-}}{\text {L.u.b. } f),}\right. \\
& \left|u_{1}\right| \leq c_{3}\left\{\underset{\Omega^{-}}{\text {l.u.b. }} g\left(x, t ; u_{0}\right)+\underset{B_{0}-}{\text { l.u.b. } \phi}+\underset{s^{-}}{\text {l.u.b. }}\left|f-B\left(x, t ; u_{0}\right)\right|\right\} .
\end{aligned}
$$

Since $g, \phi, B$ and $f$ are continuous, $u_{0}$ and $u_{1}$ are bounded. Thus we have

$$
-c_{6} \leq u_{1}<u<u_{0} \leq c_{6} \text { in } \Omega .
$$

To complete the proof of the theorem, we use the principle of mathematical induction. Let us assume that for a particular value of $i$, say $j$, we have

$$
-c_{6} \leq u_{1}<\cdots<u_{2 i+1}<u<u_{2 i}<\cdots<u_{0} \leq c_{6} \text { in } \Omega .
$$

Then for $i=j+1$, we have by (3.9) and (3.13)

$$
L\left(u_{2 j+2}-u\right)=g\left(x, t ; u_{2 i+1}\right)-g(x, t ; u)<0 \text { in } \Omega .
$$

Since $u_{2 i+2}-u=0$ on $B_{0}{ }^{-}$, and $(\partial / \partial \nu)\left(u_{2 j+2}-u\right) \geq 0$ on $S$, we have $u_{2 j+2}>u$ in $\Omega$ by an argument similar to the above. By repeating the procedure for $u_{2 i}-u_{2 j+2}$, $u-u_{2 j+3}$, and $u_{2 i+3}-u_{2 j+1}$ respectively, we obtain in $\Omega u_{2 i}>u_{2 i+2}, u>u_{2 j+3}$, and $u_{2 i+3}>u_{2 i+1}$. Thus we have (3.10).

Theorem 4. Under the hypotheses of Theorem 3 , if $u>v$ at $(y, \sigma)$ implies

$$
B(y, \sigma ; u(y, \sigma))-B(y, \sigma ; v(y, \sigma)) \leq c_{7}\{u(y, \sigma)-v(y, \sigma)\},
$$

where $c_{7}$ is a positive constant, then there exists a unique positive solution of the problem (2.1)-(2.3).

Proof. Let us rewrite the iteration scheme (3.6)-(3.8) equivalently as

$$
\begin{aligned}
u_{i+1}(x, t)= & \int_{D} N(x, t ; \xi, 0) \phi(\xi) d V_{\xi}-\int_{0}^{t} \int_{D} N(x, t ; \xi, \tau) g\left(\xi, \tau ; u_{i}\right) d V_{\xi} d \tau \\
& +\int_{0}^{t} \int_{\partial D} N(x, t ; \xi, \tau)\left[f(\xi, \tau)-B\left(\xi, \tau ; u_{i}\right)\right] d A_{\xi} d \tau .
\end{aligned}
$$

Let $\rho_{i}=\max \left|u_{i+1}-u_{i}\right|$. Then by (3.10), $\rho_{0} \leq 2 c_{6}$. Let $c_{8}=\max \left\{c_{2}, c_{7}\right\}$. By (2.6), (3.2), (3.14) and (3.15), we have

$u_{2}-u_{1} \leq c_{8}\left\{\int_{0}^{t} \int_{D} N(x, t ; \xi, \tau)\left(u_{0}-u_{1}\right) d V_{\xi} d \tau+\int_{0}^{t} \int_{\partial D} N(x, t ; \xi, \tau)\left(u_{0}-u_{1}\right) d A_{\xi} d \tau\right\}$ 
Thus

$$
\begin{aligned}
\rho_{1} & \leq c_{8} \rho_{0}\left\{\int_{0}^{t} \int_{D} N(x, t ; \xi, \tau) d V_{\xi} d \tau+\int_{0}^{t} \int_{\partial D} N(x, t ; \xi, \tau) d A_{\xi} d \tau\right\} \\
& \leq c_{8} \rho_{0}\left[\int_{0}^{t} \int_{D}\left\{q(x-\xi, t-\tau)+c_{5}\right\} d V_{\xi} d \tau+\int_{0}^{t} \int_{\partial D}\left\{q(x-\xi, t-\tau)+c_{5}\right\} d A_{\xi} d \tau\right] .
\end{aligned}
$$

Let the quantity inside the square brackets be denoted by $r . r>0$, and $\rho_{1} \leq c_{8} \rho_{0} r \leq$ $2 c_{6} c_{8} r$. It follows from induction that

$$
\rho_{n} \leq 2 c_{6}\left(c_{8} r\right)^{n} .
$$

Since $q(x-\xi, t-\tau)$ is integrable, for example by taking $\mu$ to be any arbitrarily-fixed value between $1 / 2$ and 1 , we can choose the time interval $[0, \sigma]$ such that $c_{8} r<1$ so that the sequence converges uniformly and geometrically. Thus $\lim _{i \rightarrow \infty} u_{i+1}$ is a solution of (2.1)-(2.3), and hence we have the existence of a solution on $[0, \sigma]$. Since global uniqueness of the positive solution on $\Omega^{-}$follows from Theorem 2 , we have existence of a unique positive solution on $\Omega_{0 \sigma}{ }^{-}$.

To prove the global existence on $\Omega^{-}$, we start from time $t=\sigma-\eta$, where $\eta$ is an arbitrarily chosen positive constant such that $\sigma-\eta>0$. An argument similar to the above gives the inequality

$$
c_{8}\left[\int_{\sigma-\eta}^{t} \int_{D}\left\{q(x-\xi, t-\tau)+c_{5}\right\} d V_{\xi} d \tau+\int_{\sigma-\eta}^{t} \int_{\partial D}\left\{q(x-\xi, t-\tau)+c_{5}\right\} d A_{\xi} d \tau\right]<1,
$$

restricting the time interval for existence. Since $\Omega^{-}$is cylindrical, the same length $\sigma$ of time satisfies this inequality. Thus we have a unique positive solution for $0 \leq t \leq$ $2 \sigma-\eta$. Proceeding in this way, we obtain the global existence of a unique positive solution.

4. Quadratic convergence, upper and lower bounds. We shall use the following lemma.

Lemma 2. Let $w(x, t)$ be continuous on $\Omega^{-}$, and $L w \leq 0$ in $\Omega$. (i) If $w \geq 0$ on $B_{0}{ }^{-}$ and $\psi_{\lambda} w \geq 0$ on $S$, where $\lambda(x, t)>0$, then $w \geq 0$ on $\Omega^{-}$. (ii) If $w>0$ on $B_{0}{ }^{-}$and $\psi_{\lambda} w>0$ on $S$, then $w>0$ on $\Omega^{-}$.

Proof. (i) If $w<0$ at some point of $\Omega^{-}$, then by the weak maximum principle $w$ attains its negative minimum at some point on $S$. Thus at this point $\psi_{\lambda} w<0$, contradicting the given condition $\psi_{\lambda} w \geq 0$. Thus $w \geq 0$ in $\Omega^{-}$.

(ii) If $w \leq 0$ at some point of $\Omega^{-}$, then again by the weak maximum principle $w$ attains its non-positive mininum at some point on $S$. At this point, $\psi_{\lambda} w \leq 0$, which is a contradiction to $\psi_{\lambda} w>0$. Hence $w>0$ on $\Omega^{-}$.

We shall need the following assumptions:

(B) $g$ is twice continuously differentiable in $u$ such that

$$
0 \leq g_{u} \leq c_{9}, \text { and } 0 \leq g_{u u}<\infty \text { for } u>0,
$$

where $c_{9}$ is a constant.

(C) $g_{u}(x, t ; u)$ is uniformly Hölder-continuous when $(x, t) \in \Omega^{-}$and $u$ varies in a bounded set. 
(D) $B$ is twice continuously differentiable in $u$ such that

$$
0<B_{u}<\infty \text {, and } 0 \leq B_{u u}<\infty \text { for } u>0 .
$$

Let us define a sequence $\left\{u_{i}\right\}$ by the method of quasilinearization: $u_{0}$ is any positive continuous function, conveniently given by (3.3)-(3.5), and for $i=0,1,2, \cdots$,

$$
\begin{gathered}
L u_{i+1}=g\left(x, t ; u_{i}\right)+g_{u}\left(x, t ; u_{i}\right)\left(u_{i+1}-u_{i}\right) \text { in } \Omega \\
u_{i+1}(x, 0)=\phi(x) \text { on } B_{0}{ }^{-} \\
\left(\partial u_{i+1} / \partial \nu\right)+B\left(x, t ; u_{i}\right)+B_{u}\left(x, t ; u_{i}\right)\left(u_{i+1}-u_{i}\right)=f(x, t) \text { on } S .
\end{gathered}
$$

The following theorem gives the upper bounds for the solution of the problem (2.1)(2.3).

Theorem 5. Under conditions (2.7)-(2.10), assumptions $(A)-(D)$ and $\partial D \in C^{2+\alpha}$, if $\phi$ and $f$ are continuous on $B_{0}{ }^{-}$and $S^{-}$respectively, then the sequence $\left\{u_{i}\right\}$ given by (3.3)-(3.5) and (4.3)-(4.5) is well defined, and satisfies

$$
c_{10} \geq u_{i} \geq u_{i+1}>0 \text { on } \Omega^{-}, \quad i=0,1,2, \cdots,
$$

where

$$
c_{10}=c_{3}\left(\underset{B_{0^{-}}}{\text {l.u.b. }} \phi+\underset{S^{-}}{\text {l.u.b.f }}\right) \text {. }
$$

Proof. By Taylor's theorem,

$$
g(x, t ; u)=g\left(x, t ; u_{i}\right)+g_{u}\left(x, t ; u_{i}\right)\left(u-u_{i}\right)+g_{u u}(x, t ; \eta)\left(u-u_{i}\right)^{2} / 2,
$$

where $\eta$ lies between $u$ and $u_{i}$. Since $g(x, t ; 0)=0$, and $g_{u u} \geq 0$ for $u>0$, we have at $u=0$,

$$
0 \geq g\left(x, t ; u_{i}\right)-g_{u}\left(x, t ; u_{i}\right) u_{i} \quad \text { if } \quad u_{i}>0
$$

Similarly,

$$
0 \geq B\left(x, t ; u_{i}\right)-B_{u}\left(x, t ; u_{i}\right) u_{i} \quad \text { if } \quad u_{i}>0 .
$$

First we show that $u_{i}>0(i=0,1,2, \cdots)$. If $u_{0} \leq 0$ at some point of $\Omega^{-}$, then it follows from the weak maximum principle and $\phi>0$ that $u_{0}$ attains its non-positive minimum at some point on $S$. Thus at this point $\partial u_{0} / \partial \nu \leq 0$, contradicting $f>0$. Hence $u_{0}>0$ on $\Omega^{-}$. Now we use the principle of mathematical induction. Let us assume that for a particular value of $i$, say $j, u_{i}>0$ on $\Omega^{-}$. For $i=j+1$, it follows from (4.3) and (4.7) that

$$
\left[L-g_{u}\left(x, t ; u_{i}\right)\right] u_{i+1} \leq 0 \text { in } \Omega .
$$

$u_{i+1}>0$ on $B_{0}^{-}$. From (4.5), (4.8) and $f>0$,

$$
\left[\frac{\partial}{\partial \nu}+B_{u}\left(x, t ; u_{i}\right)\right] u_{i+1}>0 \text { on } S .
$$

Since $g_{u}\left(x, t ; u_{i}\right) \geq 0$ and $B\left(x, t ; u_{i}\right)>0$, we have from Lemma 2 , where $L$ is now replaced by $L-g_{u}\left(x, t ; u_{i}\right)$, that $u_{i+1}>0$ on $\Omega^{-}$. Thus $u_{i}>0(i=0,1,2, \cdots)$ on $\Omega^{-}$.

Next we show that $u_{i} \geq u_{i+1}$. From (4.1) and (4.6),

$$
g\left(x, t ; u_{i+1}\right) \geq g\left(x, t ; u_{i}\right)+g_{u}\left(x, t ; u_{i}\right)\left(u_{i+1}-u_{i}\right) .
$$


Similarly, we have

$$
B\left(x, t ; u_{i+1}\right) \geq B\left(x, t ; u_{i}\right)+B_{u}\left(x, t ; u_{i}\right)\left(u_{i+1}-u_{i}\right) .
$$

From $g(x, t ; 0)=0, g\left(x, t ; u_{0}\right) \geq 0$, and (4.9), we have

$$
L u_{i} \leq g\left(x, t ; u_{i}\right) \text { in } \Omega
$$

for $i=0,1,2, \cdots$. Similarly, from $B(x, t ; 0)=0, B\left(x, t ; u_{0}\right)>0$, and (4.10), we obtain

$$
\left(\partial u_{i} / \partial \nu\right)+B\left(x, t ; u_{i}\right) \geq f(x, t) \text { on } S
$$

for $i=0,1,2, \cdots$. Using (4.11) and (4.12), we get

$$
\begin{array}{r}
{\left[L-g_{u}\left(x, t ; u_{i}\right)\right]\left(u_{i}-u_{i+1}\right) \leq 0 \text { in } \Omega,} \\
{\left[\frac{\partial}{\partial \nu}+B_{u}\left(x, t ; u_{i}\right)\right]\left(u_{i}-u_{i+1}\right) \geq 0 \text { on } S}
\end{array}
$$

for $i=0,1,2, \cdots$. It follows from (4.1) and (4.2) respectively that $g_{u}\left(x, t ; u_{i}\right) \geq 0$ and $B_{u}\left(x, t ; u_{i}\right)>0$. Since $u_{i}-u_{i+1}=0$ on $B_{0}{ }^{-}$, we have from Lemma 2 , where $L$ is now replaced by $L-g_{u}\left(x, t ; u_{i}\right)$, that $u_{i} \geq u_{i+1}$ on $\Omega^{-}$for $i=0,1,2, \cdots$.

By Lemma 1, $c_{10} \geq u_{0}$. Thus $c_{10} \geq u_{i} \geq u_{i+1}>0$.

Under assumptions $(A)$ and $\partial D \in C^{2+\alpha}, N(x, t ; \xi, \tau)$ exists and hence $u_{0}$ is welldefined. Since $0<u_{0} \leq c_{10}$ on $\Omega^{-}$, it follows from assumption $(C)$ that $g_{u}\left(x, t ; u_{0}\right)$ is uniformly Hölder-continuous, and hence the Neumann function $R_{1}(x, t ; \xi, \tau)$ associated with $\left[L-g_{u}\left(x, t ; u_{0}\right)\right] u_{1}=0$ in $\Omega$ and $\left[(\partial / \partial \nu)+B_{u}\left(x, t ; u_{0}\right)\right] u_{1}=0$ on $S$ exists. Thus $u_{1}$ is well-defined. By repeating the above procedures, we see that the sequence $\left\{u_{i}\right\}$ is well-defined. Thus the theorem is proved.

Theorem 6. Under the hypotheses of Theorem 5, there exists a unique positive solution of the problem (2.1)-(2.3).

Proof. $\quad c_{9} \geq g_{u} \geq 0$ implies (2.4) and (2.6). $B_{u}>0$ implies (2.5). Hence by Theorem 2 , the problem (2.1)-(2.3) has at most one positive solution.

Since the sequence $\left\{u_{i}\right\}$ is monotone non-increasing and is bounded below, there exists a function $U(x, t)$ such that $\lim _{i \rightarrow \infty} u_{i}=U$. To show $U(x, t)$ is the solution of the problem (2.1)-(2.3), let us rewrite the iteration scheme (4.3)-(4.5) equivalently as

$$
\begin{aligned}
u_{i+1}(x, t)= & \int_{D} N(x, t ; \xi, 0) \phi(\xi) d V_{\xi} \\
& -\int_{0}^{t} \int_{D} N(x, t ; \xi, \tau)\left[g\left(\xi, \tau ; u_{i}\right)+g_{u}\left(\xi, \tau ; u_{i}\right)\left(u_{i+1}-u_{i}\right)\right] d V_{\xi} d \tau \\
& +\int_{0}^{t} \int_{\partial D} N(x, t ; \xi, \tau)\left[f(\xi, \tau)-B\left(\xi, \tau ; u_{i}\right)-B_{u}\left(\xi, \tau ; u_{i}\right)\left(u_{i+1}-u_{i}\right)\right] d A_{\xi} d \tau .
\end{aligned}
$$

By (3.2) and Theorem 5, the integrands in the second and third integrals of (4.13) are bounded respectively by

$$
N(x, t ; \xi, \tau)\left[g\left(\xi, \tau ; c_{10}\right)+c_{10} g_{u}\left(\xi, \tau ; c_{10}\right)\right],
$$

and

$$
N(x, t ; \xi, \tau)\left[f(\xi, \tau)+B\left(\xi, \tau ; c_{10}\right)+c_{10} B_{u}\left(\xi, \tau ; c_{10}\right)\right]
$$


both of which are integrable over their respective regions of integration. Let us take the limit as $i$ tends to infinity in (4.13). By the Lebesgue convergence theorem [17, p. 200], we can interchange the limit and integration processes. Hence

$$
\begin{aligned}
U(x, t)= & \int_{D} N(x, t ; \xi, 0) \phi(\xi) d V_{\xi}-\int_{0}^{t} \int_{D} N(x, t ; \xi, \tau) g(\xi, \tau ; U) d V_{\xi} d \tau \\
& +\int_{0}^{t} \int_{\partial D} N(x, t ; \xi, \tau)[f(\xi, \tau)-B(\xi, \tau ; U)] d A_{\xi} d \tau .
\end{aligned}
$$

This implies that $U(x, t)$ is the solution of the problem (2.1)-(2.3).

We give another proof of existence of the solution in the next theorem. The proof also shows that the sequence $\left\{u_{i}\right\}$ converges quadratically to the solution.

Theorem 7. Under the hypotheses of Theorem 5, the problem (2.1)-(2.3) has a unique positive solution to which the sequence $\left\{u_{i}\right\}$ converges quadratically.

Proof. As in Theorem 6, uniqueness of a positive solution follows from Theorem 2. By Theorem $5, \rho_{0} \leq c_{10}$. For $i=1,2, \cdots$, we have by Taylor's theorem

$$
g\left(x, t ; u_{i}\right)=g\left(x, t ; u_{i-1}\right)+g_{u}\left(x, t ; u_{i-1}\right)\left(u_{i}-u_{i-1}\right)+g_{u u}(x, t ; \gamma)\left(u_{i}-u_{i-1}\right)^{2} / 2
$$

where $\gamma(x, t)$ lies between $u_{i}$ and $u_{i-1}$. Thus, from (4.3),

$$
L\left(u_{i}-u_{i+1}\right)=g_{u}\left(x, t ; u_{i}\right)\left(u_{i}-u_{i+1}\right)-g_{u u}(x, t ; \gamma)\left(u_{i-1}-u_{i}\right)^{2} / 2 \text { in } \Omega .
$$

Similarly, by Taylor's theorem and (4.5),

$$
\frac{\partial}{\partial \nu}\left(u_{i}-u_{i+1}\right)=-B_{u}\left(x, t ; u_{i}\right)\left(u_{i}-u_{i+1}\right)+B_{u u}(x, t ; \zeta)\left(u_{i-1}-u_{i}\right)^{2} / 2 \quad \text { on } \quad S,
$$

where $\zeta(x, t)$ lies between $u_{i}$ and $u_{i-1}$. Since $u_{i}-u_{i+1}=0$ on $B_{0}{ }^{-}, u_{i}-u_{i+1} \geq 0$ on $\Omega^{-}, N(x, t ; \xi, \tau)>0$ on $\Omega_{\tau T}, g_{u} \geq 0$ and $B_{u}>0$, we have for $i=1,2,3, \cdots$,

$$
\begin{aligned}
u_{i}-u_{i+1} \leq & \int_{0}^{t} \int_{D} N(x, t ; \xi, \tau) g_{u u}(\xi, \tau ; \gamma)\left(u_{i-1}-u_{i}\right)^{2} / 2 d V_{\xi} d \tau \\
& +\int_{0}^{t} \int_{\partial D} N(x, t ; \xi, \tau) B_{u u}(\xi, \tau ; \zeta)\left(u_{i-1}-u_{i}\right)^{2} / 2 d A_{\xi} d \tau
\end{aligned}
$$

by dropping out the non-positive terms. Since $u_{i} \leq c_{10}$, let

$$
c_{11}=\max \left\{\underset{\Omega^{-} \times\left[0, c_{10}\right]}{\text { l.u.b. }} g_{u u}(x, t ; u), \underset{S-\times\left[0, c_{10}\right]}{\text { l.u.b. }} B_{u u}(x, t ; u)\right\} .
$$

Thus

$$
\rho_{i} \leq c_{11} \rho_{i-1}^{2}\left\{\int_{0}^{t} \int_{D} N(x, t ; \xi, \tau) d V_{\xi} d \tau+\int_{0}^{t} \int_{\partial D} N(x, t ; \xi, \tau) d A_{\xi} d \tau\right\} / 2 .
$$

Following the proof of Theorem 4, we have

$$
\rho_{i} \leq \rho_{i-1}^{2}\left(c_{11} r\right) / 2 \text { for } i=1,2, \cdots .
$$

Let us choose the time interval $[0, \delta]$ such that $c_{11} r / 2<1$. Then the sequence converges quadratically and uniformly on $[0, \delta]$.

An argument similar to the proof of Theorem 4 establishes the global existence of the positive solution on $\Omega^{-}$. 
To construct a bounded non-decreasing sequence, we shall use the following assumption:

(E) $g$ and $B$ are continuously differentiable in $u$, and there exists a bounded uniformly Hölder-continuous function $\theta(x, t)$ on $\Omega^{-}$such that

$$
\theta(x, t) \geq g_{u}(x, t ; u) \geq 0
$$

and a continuous function $s(x, t)$ on $S^{-}$such that

$$
s(x, t) \geq B_{u}(x, t ; u)>0 .
$$

We note that in particular $\theta$ and $s$ can be replaced by appropriate constants. Let us construct a sequence $\left\{v_{i}\right\}: v_{0} \equiv 0$, and $v_{i+1}(i=0,1,2, \cdots)$ are given by

$$
\begin{gathered}
L v_{i+1}=g\left(x, t ; v_{i}\right)+\theta(x, t)\left(v_{i+1}-v_{i}\right) \text { in } \Omega \\
v_{i+1}(x, 0)=\phi(x) \text { on } B_{0}^{-}, \\
\left(\partial v_{i+1} / \partial \nu\right)+B\left(x, t ; v_{i}\right)+s(x, t)\left(v_{i+1}-v_{i}\right)=f(x, t) \text { on } S .
\end{gathered}
$$

Under assumptions $(A),(E)$ and $\partial D \in C^{2+\alpha}$, the Neumann function of $(L-\theta) w=0$ in $\Omega$ and $\psi_{s} w=0$ on $S$ exists, and hence the sequence $\left\{v_{i}\right\}$ is well defined.

Theorem 8. Under the hypotheses of Theorem 5 with assumptions $(B),(C)$ and $(D)$ replaced by assumption $(E)$, the sequence $\left\{v_{i}\right\}$ satisfies

$$
0=v_{0}<v_{1} \leq v_{2} \leq \cdots \leq u_{0} \leq c_{10} \text { on } \Omega^{-},
$$

where $c_{10}=c_{3}$ (l.u.b. B $_{0}-\phi+$ l.u.b. $s^{-} f$ ).

Proof. $v_{1}$ satisfies $(L-\theta) v_{1}=0$ in $\Omega, v_{1}>0$ on $B_{0}{ }^{-}, \psi_{s} v_{1}>0$ on $S$. By Lemma 2, $v_{1}>0$ on $\Omega^{-}$. Let us assume that for a particular value of $i$, say $j(\geq 1)$, we have $v_{0}<$ $v_{1} \leq v_{2} \leq \cdots \leq v_{i}$. Then, from (4.16),

$$
(L-\theta)\left(v_{i+1}-v_{i}\right)=g\left(x, t ; v_{i}\right)-g\left(x, t ; v_{i-1}\right)-\theta\left(v_{i}-v_{i-1}\right) .
$$

By the mean value theorem and (4.14),

$$
g\left(x, t ; v_{i}\right)-g\left(x, t ; v_{i-1}\right) \leq \theta\left(v_{i}-v_{i-1}\right) .
$$

Therefore $(L-\theta)\left(v_{i+1}-v_{i}\right) \leq 0$ in $\Omega$. Similarly, from (4.15) and (4.18), $\psi_{\text {( }}\left(v_{i+1}-v_{i}\right) \geq 0$ on $S$. From (4.17), $v_{i+1}-v_{i}=0$ on $B_{0}^{-}$. From Lemma $2, v_{i+1} \geq v_{i}$ on $\Omega^{-}$. Thus $v_{0}<$ $v_{1} \leq v_{2} \leq \cdots$.

Since $v_{i} \geq v_{i-1}, g(x, t ; w) \geq 0$ and $B(x, t ; w)>0$ for $w>0$, we have $L\left(u_{0}-v_{i}\right) \leq 0$ in $\Omega$, and $(\partial / \partial \nu)\left(u_{0}-v_{i}\right)>0$ on $S$ for $i \geq 2 . u_{0}-v_{i}=0$ on $B_{0}{ }^{-}$. Thus, by the weak maximum principle, $u_{0} \geq v_{i}$ on $\Omega^{-}$for $i \geq 2$. From Lemma $1, u_{0} \leq c_{10}$.

The following theorem shows that the sequence $\left\{v_{i}\right\}$ forms the lower bounds to the solution. Its proof is similar to that for Theorem 6 , and hence is omitted.

Theorem 9. Under the hypotheses of Theorem 8 , there exists a unique positive solution $\lim _{i \rightarrow \infty} v_{i}$ of the problem (2.1)-(2.3).

For the scheme (4.16)-(4.18), the same Neumann function corresponding to $(L-\theta) w=0$ and $\psi_{s} w=0$ occurs in all steps in the construction of the solution. Let us rewrite (4.3) and (4.5) respectively as

$$
\begin{gathered}
{\left[L-g_{u}\left(x, t ; u_{i}\right)\right] u_{i+1}=g\left(x, t ; u_{i}\right)-g_{u}\left(x, t ; u_{i}\right) u_{i} \text { in } \Omega,} \\
{\left[\frac{\partial}{\partial \nu}+B_{u}\left(x, t ; u_{i}\right)\right] u_{i+1}=f-B\left(x, t ; u_{i}\right)+B_{u}\left(x, t ; u_{i}\right) u_{i} \quad \text { on } \quad S .}
\end{gathered}
$$


$g_{u}\left(x, t ; u_{i}\right)$ and $B_{u}\left(x, t ; u_{i}\right)$ vary as $i$ varies. Hence the associated Neumann function $R_{i+1}(x, t ; \xi, \tau)$ varies in each successive step of the construction in the quasilinearization technique. Although the rate of convergence is geometrical in the Picard scheme (3.3)(3.8), the Neumann function $V(x, t ; \xi, \tau)$ remains the same in all steps.

\section{REFERENCES}

[1] R. Bellman, Functional equations in the theory of dynamics programming. V: Positivity and quasilinearity, Proc. Nat. Acad. Sci. U.S.A. 41, 743-746 (1955)

[2] C. Y. Chan, A nonlinear second initial boundary value problem for the heat equation, Quart. Appl. Math. 29, 261-268 (1971)

[3] D. S. Cohen, Generalized radiation cooling of a convex solid, J. Math. Anal. Appl. 35, 503-511 (1971)

[4] D. S. Cohen and T. W. Laetsch, Nonlinear boundary value problems suggested by chemical reactor theory, J. Diff. Eqs. 7, 217-226 (1970)

[5] A. Friedman, Generalized heat transfer between solids and gases under nonlinear boundary conditions, J. Math. Mech. 8, 161-183 (1959)

[6] A. Friedman, Partial differential equations of parabolic type, Prentice-Hall, Englewood Cliffs, N. J., 1964 , pp. 3-25, 34-40, 44-45, 82-84, 134, 146, 155

[7] S. Itô, A boundary value problem of partial differential equations of parabolic type, Duke Math. J. 24, 299-312 (1957)

[8] S. Itô, A remark on my paper "A boundary value problem of partial differential equations of parabolic type" in Duke Mathematical Journal, Proc. Japan Acad. 34, 463-465 (1958)

[9] II. B. Keller, Elliptic boundary value problems suggested by nonlinear diffusion processes, Arch. Rat. Mech. Anal. 35, 363-381 (1969)

[10] J. B. Keller and W. E. Olmstead, Temperature of a nonlinearly radiating semi-infinite solid, Quart. Appl. Math. 29, 559-566 (1972)

[11] W. R. Mann and F. Wolf, Heat transfer between solids and gases under nonlinear boundary conditions, Quart. Appl. Math. 9, 163-184 (1951)

[12] L. Nirenberg, A strong maximum principle for parabolic equations, Comm. Pure Appl. Math. 6, $167-177$ (1953)

[13] W. E. Olmstead, Temperalure distribution in a convex solid with a nonlinear radiation boundary condition, J. Math. Mech. 15, 899-907 (1966)

[14] K. Padmavally, On a non-linear integral equation, J. Math. Mech. 7, 533-555 (1958)

[15] M. H. Protter and H. F. Weinberger, Maximum principles in differential equations, Prentice-IIall, Englewood Cliffs, N. J., 1967, pp. 173-175

[16] J. I. Roberts and W. R. Mann, On a certain nonlinear integral equation of the Vollerra type, Pacific J. Math. 1, 431-445 (1951)

[17], I. L. Royden, Real analysis, Macmillan, N. Y., 1963, p. 200

[18] J. Schauder, Der Fixpunktsatz in Funktionalräumen, Studia Math. 2, 171-180 (1930) 